\title{
Diferencias de género y edad en autoconcepto en estudiantes adolescentes chilenos
}

\author{
Gender and age differences in self-concept in Chilean \\ adolescent students
}

\author{
María Vicent ${ }^{\mathrm{a}}$, Nelly Lagos-San Martín ${ }^{\mathrm{b}}$, Carolina Gonzálvez ${ }^{\mathrm{a}}$, \\ Cándido J. Inglés ${ }^{c}$, José Manuel García-Fernández y Nieves Gomis ${ }^{\mathrm{a}}$ \\ ${ }^{a}$ Universidad de Alicante, Alicante, España ${ }^{b}$ Universidad del Bío-Bío, Chillán, Chile \\ ${ }^{c}$ Universidad Miguel Hernández, Elche, España
}

\begin{abstract}
Resumen: Investgaciones previas han puesto de manifiesto la importancia del estudio del autoconcepto durante la adolescencia. El objetivo de este estudio consistió en analizar las diferencias de género y edad en las distintas dimensiones del autoconcepto en una muestra de 1414 estudiantes chilenos de entre 13 y 18 años. El autoconcepto fue evaluado mediante la versión breve del SDQII. Los resultados evidenciaron diferencias de género para las dimensiones Autoconcepto Verbal, Apariencia Física, Sinceridad/Veracidad y Autoestima en favor de las jóvenes, así como diferencias en el Autoconcepto Matemático, Habilidades Físicas, Estabilidad Emocional y Relación con los Padres en favor de los varones. También se observó una tendencia en los alumnos de mayor edad a puntuar más alto en Autoconcepto Académico General, Habilidades Físicas, Apariencia Física, Relaciones con el Sexo Opuesto y Autoestima, que sus iguales de menor edad. Este mismo patrón, pero a la inversa, se obtuvo para las dimensiones de Autoconcepto Matemático, Verbal y Estabilidad Emocional. Los resultados no replicaron la tendencia de los varones, reportada en estudios previos, a valorar más positivamente su físico y a poseer una autoestima más elevada que las mujeres, así como a percibir más negativamente sus relaciones paterno-filiales.
\end{abstract}

Palabras clave: autoconcepto, adolescentes, diferencias de género, diferencias de edad.
Abstract: Previous researches have highlighted the importance of the study of self-concept in adolescence. The aim of this study was to analyse gender and age differences in several dimensions of the self-concept. The sample included 1414 Chilean students between 13 and 18 years old. The self-concept was assessed using the SDQII-Short form. Results revealed that girls scored significantly higher in Verbal, Physical Appearance, Honesty-Trustworthiness and Self-esteem self-concepts, whereas boys got higher scores in Maths, Physical Ability, Emotional Stability and Parents Relations self-concepts. It was also observed that older students scored higher in general Academic Self-concept, Physical Ability, Physical Appearance, Opposite Sex Relations and Self-esteem than their younger peers. This same pattern, but the other way round, was obtained for Maths, Verbal and Emotional Stability dimensions. The outcomes did not argue the tendency for men, reported in previous studies, to value more positively their physical appearance and to own higher self-esteem than women, as well as perceive more negatively their parentchild relationships.

Keywords: self-concept, adolescents, gender differences, age differences.

Parte de esta investigación ha sido financiada por el Proyecto MECESUP UBB0704-D2011 concedido a Nelly Lagos-San Martín. 
Contacto: M. Vicent, Departamento de Psicología Evolutiva y Didáctica, Facultad de Educación, Universidad de Alicante, Campus de Sant Vicent del Raspeig Ap. 99. E-03080, Alicante, España. Correo electrónico: maria.vicent@ua.es

Cómo citar: Vicent, M., Lagos-San Martín, N., Gonzálvez, C., Inglés, C. J., García-Fernández, J. M. y Gomis, N. (2015). Diferencias de género y edad en autoconcepto en estudiantes adolescentes chilenos. Revista de Psicología, 24(1), 1-16. http://dx.doi.org/10.5354/0719-0581.2015.36752 


\section{Introducción}

El autoconcepto es definido como las percepciones que una persona tiene de sí misma, conformadas a partir de las propias experiencias e influenciadas por los refuerzos y las evaluaciones de otras personas significativas, considerándose como un constructo organizado, multidimensional, jerárquico, estable, desarrollable, evaluativo y diferenciable (Shavelson, Hubner y Stanton, 1976). Basándose en esta concepción, Marsh (1992) desarrolló el Self-Description Questionnaire II (SDQII) y su versión breve SDQII-S (Marsh, Ellis, Parada, Richards y Heubeck, 2005), con objeto de evaluar este constructo en la población adolescente. Empleando dichas medidas de evaluación, se ha demostrado que el autoconcepto es un correlato negativo de problemas de internalización (Delgado, Inglés y García-Fernández, 2013; Marsh, Parada y Ayotte, 2004; Nishikawa, Hägglöf y Sundbom, 2010; Rigoli, Piek y Kane, 2012) y consumo de drogas (Vasconcelos-Rasposo, Goçalves, Teixeira y Fernandes, 2009), además de ejercer una influencia positiva en el rendimiento académico (Marsh y Hau, 2004; Massanero y Vázquez, 2005), en la conducta prosocial (Inglés, Marínez-González, GarcíaFernández, Torregrosa y Ruiz-Esteban, 2012) y en la satisfacción con la vida (Huebner, Gilman y Laughlin, 1999). Precisamente, los cambios acontecidos durante la adolescencia, como el desarrollo de las habilidades cognitivas y físicas y la aparición de nuevos roles sociales y académicos, los cuales permiten que el autoconcepto alcance un mayor nivel de abstracción y el surgimiento de nuevos dominios de autovaloración, así como la presencia de una mayor vulnerabilidad a distorsionar la autoimagen, justifican la investigación del autoconcepto en esta etapa del ciclo vital (Inglés et al., 2012).
Diferencias de género y edad en autoconcepto durante la adolescencia

Durante los últimos años, las diferencias de género en autoconcepto han sido ampliamente investigadas en la población adolescente española (Amezcua y Pichardo, 2000; Cardenal y Díaz, 2000; Garaigordobil, Durá y Pérez, 2005; GómezVela, Verdugo y González-Gil, 2007; Gordillo, Vicente, Sánchez, Gómez y Gordillo, 2011; Infante et al., 2002; Inglés et al., 2009; Padilla, García y Suárez, 2010; Pastor, Balaguer y García-Merita, 2003), mexicana (Guido, Mujica y Gutiérrez, 2011), estadounidense (Cunningham y Rinn, 2007), australiana (Hay, 2000), sueca (Nishikawa, Norlanden, Fransson y Sundbom, 2007) y asiática (Chu, 2002; Razali, Razali, Azizan y Pillai, 2014; Yun, 2001).

Centrándose en los resultados obtenidos en relación con las dimensiones evaluadas por el SDQII, Hay (2000) obtuvo que los alumnos de sexo masculino puntuaron significativamente más alto en Apariencia Física, Habilidad Física, Autoconcepto Académico General, Estabilidad Emocional y Autoconcepto Total que sus compañeras, en una muestra de 128 alumnos australianos de entre 14 y 15 años, con problemas persistentes de conducta, matriculados en un programa escolar alternativo. Yun (2001), empleando una muestra de 208 participantes chinos de $10^{\circ}$ grado (15 años de edad) compuesta por alumnos de escuelas para altas capacidades y alumnos de centros regulares, halló una tendencia en las mujeres a puntuar significativamente más alto que los varones en Autoconcepto Verbal, independientemente de la capacidad o el centro de asistencia. Por otro lado, los varones puntuaron significativamente más alto que las mujeres en Autoconcepto Matemático y Autoestima, con efectos de interacción género 
por tipo de escuela para ambas variables, así como para el Autoconcepto Académico General, cuyas puntuaciones fueron significativamente superiores en las mujeres que en los varones de escuelas para altas habilidades y a la inversa en los alumnos de escuelas regulares.

Por su parte, en una muestra de 591 taiwaneses de entre 15 y 17 años, Chu (2002) encontró diferencias significativas en función del género en las dimensiones de Autoconcepto Matemático, Estabilidad Emocional y Habilidades Físicas, a favor de los varones, así como en Sinceri$\mathrm{dad} /$ Veracidad, a favor de las adolescentes. También halló un efecto de interacción género/edad en las Relaciones Personales, de manera que los varones de $10^{\circ}$ grado puntuaron más alto que el resto, mientras que en el caso de las jóvenes, obtuvieron mayores puntuaciones las de $9^{\circ}$ curso. Posteriormente, Nishikawa et al. (2007) encontraron diferencias de género en las dimensiones de Apariencia Física y Relaciones con el Sexo Opuesto, con niveles más altos para los alumnos y en Sinceridad/Veracidad, con niveles más altos para las mujeres, en una muestra compuesta por 240 japoneses y suecos con edades comprendidas entre 14 y 15 años. Con una muestra de 2267 alumnos españoles de $1^{\circ}$ a $4^{\circ}$ curso de Educación Secundaria Obligatoria (ESO), con un rango de edad de entre 12 y 16 años, Inglés et al. (2009), encontraron que los varones puntuaron significativamente más alto que sus compañeras en Habilidades Físicas, Apariencia Física, Estabilidad Emocional y Matemáticas. Por otro lado, las mujeres puntuaron más en Relaciones con el Mismo Sexo, Relaciones con los Padres, Sinceridad/Veracidad y Autoconcepto Verbal. Los resultados de las regresiones logísticas revelaron que el género predijo significativamente altas puntuaciones en todas las dimensiones del auto- concepto, excepto en Relaciones con el Sexo Opuesto, Académico General y Autoconcepto Total.

En cuanto a las diferencias en los niveles de autoconcepto en función de la edad o el curso académico, Marsh (1989), a través de un metaanálisis, encontró que las puntuaciones en Apariencia Física, Habilidades Físicas, Relaciones con el Mismo Sexo, Sinceridad/Veracidad, Autoestima, Matemáticas y Autoconcepto Académico General, fueron superiores en $7^{\circ}$ grado (12 a 13 años), produciéndose un descenso en $8^{\circ}$ y $9^{\circ}$ grado (13 a 15 años) y un aumento en los cursos de $10^{\circ}$ a $11^{\circ}(15$ a 17 años). En esta misma línea, Chu (2002) halló que los estudiantes de $9^{\circ}$ curso (14 a 15 años) presentaron puntuaciones significativamente más altas en Estabilidad Emocional que sus compañeros de $10^{\circ}$ y $11^{\circ}$ curso (15 a 17 años). Además, los estudiantes de $10^{\circ}$ curso (15 a 16 años) mostraron puntuaciones más elevadas en las dimensiones de Matemáticas y Académico General que sus compañeros de $9^{\circ}$ (14 a 15 años) y $11^{\circ}$ (16 a 17 años). Posteriormente, en el estudio realizado por Inglés et al. (2009), también se encontraron variaciones de acuerdo con la edad en algunas variables del autoconcepto, informándose de diferencias estadísticamente significativas en Autoconcepto Académico General y Autoconcepto Total, a favor de los estudiantes de $1^{\circ}$ de ESO (12 a 13 años) respecto de los estudiantes de $3^{\circ}$ (14 a 15 años), y resultados inversos en la variable Relaciones con los Padres entre ambos cursos. Además, se encontraron diferencias entre los ciclos educativos de educación secundaria para la variable Verbal, señalándose que los estudiantes del primer ciclo de ESO $\left(1^{\circ}\right.$ y $2^{\circ}$ curso, entre 12 y 14 años) presentaron puntuaciones significativamente más altas que sus compañeros de segundo ciclo ( $3^{\circ}$ y $4^{\circ}$ curso, entre 14 y 16 años), 
mientras que para la dimensión Relaciones con el Sexo Opuesto, los alumnos de $1^{\circ}$ y $2^{\circ}$ (12 a 14 años) puntuaron significativamente más bajo que los alumnos de $4^{\circ}$ curso de ESO (15 a 16 años). Igualmente, las puntuaciones de los estudiantes de $1^{\circ}$ (12 a 13 años) fueron estadísticamente superiores a las del alumnado de $2^{\circ}$ (13 a 14 años) y significativamente más bajas que los de $4^{\circ}$ (15 a 16 años) para la dimensión Apariencia Física. Similarmente, los análisis de regresión logística también evidenciaron que el curso académico fue un predictor significativo del Autoconcepto Total y de las dimensiones Relaciones con el Sexo Opuesto, Apariencia Física, Sinceridad/Veracidad, Verbal y Académico General.

En síntesis, desde nuestro conocimiento, ningún estudio ha examinado las diferencias de género y edad en una población adolescente chilena. Asimismo, se desconoce la existencia de estudios que hayan examinado dichas diferencias empleando la versión breve del SDQII. Por tanto, teniendo en cuenta las limitaciones mencionadas, el presente trabajo tiene por objetivo analizar las diferencias de género $\mathrm{y}$ edad en las dimensiones que componen el autoconcepto, empleando el SDQII-S (Marsh et al., 2005) en una muestra de estudiantes chilenos de educación media, con el propósito de comprobar si los resultados obtenidos en las investigaciones previas son generalizables a esta muestra.

De este modo, en consonancia con la investigación previa realizada con el SDQII, se espera que los varones presenten puntuaciones significativamente más altas en las subescalas de Autoconcepto Matemático (Chu, 2002; Inglés et al., 2009; Yun, 2001), Apariencia Física (Hay, 2000; Inglés et al., 2009; Nishikawa et al., 2007), Habilidades Físicas y Estabilidad Emocional (Chu, 2002; Hay, 2000; Inglés et al., 2009).
Por el contrario, se espera que las adolescentes presenten puntuaciones más elevadas en las subescalas de Autoconcepto Verbal (Inglés et al., 2009; Yun, 2001) y Sinceridad/Veracidad (Chu, 2002; Inglés et al., 2009; Nishikawa et al., 2007).

Además, no se espera hallar diferencias significativas entre ambos sexos para las variables Académica General (Inglés et al., 2009; Nishikawa et al., 2007; Yun, 2001), Relación con el Sexo Opuesto (Hay, 2000; Inglés et al., 2009), Relaciones con el Mismo Sexo (Hay, 2000; Nishikawa et al., 2007), Relaciones con los Padres (Chu, 2002; Hay, 2000; Nishikawa et al., 2007), Autoestima (Hay, 2000; Nishikawa et al., 2007) y Autoconcepto Total (Inglés et al., 2009; Nishikawa et al., 2007). Por último, cabe esperar la obtención de diferencias significativas en las dimensiones del autoconcepto en función de la edad (Chu, 2002; Inglés et al., 2009; Marsh, 1989).

\section{Metodología}

\section{Participantes}

La población de referencia fue de estudiantes adolescentes chilenos. Se seleccionaron por conveniencia 10 comunas de la Provincia de Nuble, en Chile, bajo criterio de accesibilidad, y se seleccionaron al azar dos centros educativos de enseñanza media en cada una de ellas. Los centros fueron mayoritariamente de dependencia municipal y, en menor medida, particulares subvencionados, todos ellos característicos de un nivel socioeconómico y cultural medio. Posteriormente, se escogieron de forma aleatoria cuatro aulas en cada uno de los centros, una por cada curso académico. Siguiendo este procedimiento se reclutaron 1578 participantes, de los cuales 164 fueron excluidos por errores u omisiones en las respuestas en el 
cuestionario y por no obtener el consentimiento de los padres para participar en esta investigación.

La muestra final estuvo formada por 1414 estudiantes chilenos de educación media, 695 hombres y 719 mujeres, con edades comprendidas entre los 13 y 18 años $(M=$ $15.31 ; D E=1.45)$. La distribución por género y edad de los participantes se puede observar en la tabla 1. La Prueba Chicuadrado de homogeneidad de la distribución de frecuencias reveló la ausencia de diferencias estadísticamente significativas entre los seis grupos de sexo por edad, $\chi^{2}$ $(2, N=1414)=2.39, p=.30$.

\section{Instrumento}

Se utilizó la versión breve del SelfDescription Questionnaire, SDQII-S (Marsh et al., 2005) adaptada al castellano por García-Fernández et al. (2006). Se trata de un autoinforme que mide el autoconcepto en adolescentes de 12 a 18 años. Contiene un total de 51 ítems, ubicados en una escala Likert de 6 puntos $(1=$ falso a $6=$ verdadero), y distribuidos en 11 dimensiones de las cuales tres son académicas (General, Matemáticas y Verbal), siete no académicas (Apariencia Física, Habilidades Físicas, Relaciones con el Sexo Opuesto, Relaciones con el Mismo Sexo, Relación con los Padres, Sinceridad/Veracidad y Estabilidad Emocional) y una de Autoestima. Los niveles de consistencia interna para este trabajo fueron aceptables (véase la tabla 2), aunque ligeramente inferiores a los obtenidos en el estudio realizado por Marsh et al. (2005), los cuales oscilaron entre .80 y .89 .

Lagos-San Martín, García-Fernández, Inglés, Gómez-Núñez e Hidalgo (en prensa), evaluaron las propiedades psicométricas de la versión breve del SDQII en una muestra de 1255 estudiantes chilenos de entre 13 y 17 años. El análisis factorial confirmatorio ratificó la estructura de 11 factores correlacionados, con cargas factoriales superiores a .30 para todos los ítems e intercorrelaciones que oscilaron entre .03 y .07. Los niveles de Alfa de Cronbach oscilaron entre .84 (Relaciones con los Padres) y .70 (Autoestima). Se hallaron correlaciones positivas y significativas entre las 11 dimensiones y la Escala de Autoeficacia Académica Percibida de Situaciones Académicas (Palenzuela, 1983), excepto para la dimensión Estabilidad Emocional, cuyas puntuaciones no alcanzaron la significación estadística. Asimismo, la validez discriminante de la escala fue apoyada por los resultados de los análisis correlacionales entre las dimensiones del SDQII-S y el Inventario de Ansiedad Estado-Rasgo (Spielberger, Gorsuch y Lushene, 1970), los cuales mostraron asociaciones negativas y significativas en la mayoría de los casos.

Tabla 1

Distribución de participantes según el género y la edad

\begin{tabular}{lcccc} 
& $13-14$ años & $15-16$ años & $17-18$ años & \\
\cline { 2 - 4 } & $N(\%)$ & $N(\%)$ & $N(\%)$ & Total $(\%)$ \\
\cline { 2 - 4 } Hombres & $217(15.3)$ & $296(20.9)$ & $182(12.9)$ & $695(49.2)$ \\
Mujeres & $199(14.1)$ & $330(23.3)$ & $190(13.4)$ & $719(50.8)$ \\
Total & $416(29.4)$ & $626(44.3)$ & $372(26.3)$ & $1414(100)$ \\
\hline
\end{tabular}


Tabla 2

Descripción y consistencia interna de las subescalas del SDQII-S

\begin{tabular}{|c|c|c|c|}
\hline Subescala & Descripción & $\begin{array}{c}N \\
\text { ítems } \\
\end{array}$ & $\begin{array}{l}\text { Alfa de } \\
\text { Cronbach }\end{array}$ \\
\hline Académico General & $\begin{array}{l}\text { Habilidad, disfrute e interés por las asigna- } \\
\text { turas escolares (e.g., "Aprendo las cosas con } \\
\text { rapidez en la mayoría de las asignaturas"). }\end{array}$ & 4 & .77 \\
\hline Matemáticas & $\begin{array}{l}\text { Habilidad, disfrute e interés en matemáti- } \\
\text { cas y razonamiento (e.g., "Matemáticas es } \\
\text { una de mis asignaturas favoritas"). }\end{array}$ & 4 & .83 \\
\hline Verbal & $\begin{array}{l}\text { Habilidad, disfrute e interés en lengua y } \\
\text { lectura (e.g., "Aprendo las cosas con rapi- } \\
\text { dez en las clases de Lengua"). }\end{array}$ & 5 & .79 \\
\hline Apariencia Física & Atractivo físico (e.g., "Soy guapo/a"). & 4 & .77 \\
\hline Habilidades Físicas & $\begin{array}{l}\text { Habilidades e intereses por los deportes y } \\
\text { las actividades físicas (e.g., "Me gustan } \\
\text { las cosas como los deportes, la gimnasia y } \\
\text { bailar"). }\end{array}$ & 4 & .82 \\
\hline Estabilidad Emocional & $\begin{array}{l}\text { Bienestar emocional y ausencia de psico- } \\
\text { patologías (e.g., "Me enfado con facili- } \\
\text { dad"). }\end{array}$ & 5 & .74 \\
\hline $\begin{array}{l}\text { Relaciones con los } \\
\text { Padres }\end{array}$ & $\begin{array}{l}\text { Interacciones con los padres (e.g., "Me } \\
\text { llevo bien con mis padres"). }\end{array}$ & 4 & .84 \\
\hline $\begin{array}{l}\text { Relaciones con el Sexo } \\
\text { Opuesto }\end{array}$ & $\begin{array}{l}\text { Interacciones con personas del sexo } \\
\text { opuesto (e.g., "No soy muy popular entre } \\
\text { las personas del sexo opuesto"). }\end{array}$ & 4 & .73 \\
\hline $\begin{array}{l}\text { Relaciones con el } \\
\text { Mismo Sexo }\end{array}$ & $\begin{array}{l}\text { Interacciones con personas del mismo } \\
\text { sexo (e.g., "Me resulta difícil hacer ami- } \\
\text { gos de mi mismo sexo"). }\end{array}$ & 5 & .72 \\
\hline Sinceridad/Veracidad & $\begin{array}{l}\text { Sinceridad y dependencia (e.g., "Soy sin- } \\
\text { cero/a"). }\end{array}$ & 6 & .71 \\
\hline Autoestima & $\begin{array}{l}\text { Autovalía, confianza en uno mismo y sa- } \\
\text { tisfacción con uno mismo (e.g., "En gene- } \\
\text { ral soy un/a fracasado/a"). }\end{array}$ & 6 & .70 \\
\hline
\end{tabular}

La versión corta del SDQII (Marsh et al., 2005) ha sido validada y/o utilizada en población adolescente australiana (Bodkin-Andrews, Hay, Craven y Yeung, 2010; Bodkin-Andrews, O'Rourke, Dillon, Graven y Yeung, 2012; Marsh, Martin y Jack- son, 2010; Simmons y Hay, 2010), eslovaca (Grum, 2006), japonesa (Nishikawa et al., 2010; Nishikawa, Sundbom y Hägglöf, 2010) y española (García-Fernández et al., 2006), reportando unas propiedades psicométricas adecuadas. 


\section{Procedimiento}

Al iniciar el estudio se realizó una reunión con los equipos directivos de los centros seleccionados para exponer los objetivos de la investigación, solicitar la autorización necesaria y coordinar el horario para la administración de los instrumentos. Posteriormente, de forma previa al consentimiento informado por parte de los padres, se aplicaron los cuestionarios colectivamente y durante el horario lectivo, estando presente en todo momento un investigador para explicar la forma de responder al test, enfatizando la importancia de contestar de manera independiente y veraz cada ítem, aclarando dudas y verificando el correcto cumplimiento de los datos de identificación (e.g., número de participante, edad, sexo y centro, entre otros). Los participantes fueron tratados en todo momento según los criterios éticos que rigen la investigación científica. Para garantizar el anonimato de los datos, se asignó a cada alumno un número arbitrario que únicamente conocía el sujeto. Finalmente, se agradeció la participación y colaboración prestada a los estudiantes, a los profesores que acompañaron a los investigadores durante la aplicación y a los directores, entregándoles un informe con los resultados obtenidos para cada centro educativo.

\section{Análisis de datos}

Se realizó un análisis de varianza ANOVA $2 \times 3$ para determinar las diferencias de acuerdo al género y a la edad en cada una de las dimensiones del autoconcepto contenidas en el SDQII-S y el efecto de la interacción entre ambas variables. Para identificar entre qué edades se hallaban las diferencias se llevaron a cabo comparaciones post hoc (Prueba de Scheffé). Además, se calculó el tamaño del efecto o diferencia de media tipificada (índice $d$ ), el cual fue interpretado de acuerdo a lo planteado por Cohen (1988), quien señala que los valores entre .20 y .49 indican un tamaño del efecto pequeño; entre $.50 \mathrm{y}$ .79 un tamaño del efecto medio o moderado; y valores iguales o mayores a .80 serían señal de un gran tamaño.

\section{Resultados}

Los resultados indicaron que las jóvenes presentaron puntuaciones significativamente más altas que los varones en las escalas de Autoconcepto Verbal, Apariencia Física, Sinceridad/Veracidad y Autoestima. Los varones, en cambio, presentaron puntuaciones significativamente superiores en Matemáticas, Habilidades Físicas, Estabilidad Emocional y Relaciones con los Padres. No se encontraron diferencias estadísticamente significativas en el resto de dimensiones del SDQII-S. Los tamaños del efecto fueron de baja magnitud en todos los casos, exceptuando la escala Estabilidad Emocional cuyo tamaño del efecto fue moderado $(d=.59)$ (véase la tabla 3).

En relación con la edad, los resultados mostraron diferencias estadísticamente significativas en todas las dimensiones del autoconcepto analizadas, excepto en Relaciones con los Padres, Relaciones con el Mismo Sexo y Sinceridad/Veracidad (véase la tabla 4). En concreto, se observó que para las subescalas de Autoconcepto Académico General, Apariencia Física, Habilidades Físicas, Relaciones con el Sexo Opuesto y Autoestima, las puntuaciones tendían a aumentar con la edad; por el contrario, los participantes de mayor edad (17-18 años), obtuvieron puntuaciones más bajas que sus iguales de menor edad para las dimensiones de autoconcepto en Matemáticas, Verbal y Estabilidad Emocional. En este sentido, la Prueba Scheffé reveló que 
los alumnos de entre 17 y 18 años, puntuaron más alto en Apariencia Física que sus compañeros de entre 15 y $16(d=.36)$ y 13 y 14 años $(d=.23)$. Similarmente, los sujetos de mayor edad, obtuvieron una media más alta en Autoestima que sus iguales de entre 15 y 16 años $(d=.24)$ y 13 y 14 años $(d=.38)$. También fueron significativas las diferencias encontradas entre los alumnos con 15 y 16 años y de entre 13 y $14(d=.15)$. Respecto de la dimensión Habilidad Física, los alumnos de 17 y 18 años puntuaron significativamente más alto que aquellos de entre $13 \mathrm{y}$ 14 años $(d=.35)$ y de entre 15 y 16 años $(d=.30)$. Este mismo patrón se cumplió para la dimensión Autoconcepto Académico General $(d=.19$, para las diferencias de entre 17 y 18 y de entre 13 y 14 años y $d=.19$, para las diferencias entre 17 y 18 años y entre 15 y 16). En cuanto a las Relaciones con el Sexo Opuesto, únicamente fueron significativas las diferencias entre los alumnos de 17 y 18 años y los de 13 y $14(d=.18)$.
Por el contrario, se halló que los sujetos de entre 13 y 14 años puntuaron significativamente más alto en Estabilidad Emocional que el grupo de mayor edad $(d=.22)$ y el de 15 y 16 años $(d=.29)$. El mismo patrón de diferencias se encontró para el Autoconcepto Matemático $(d=.16$, para las diferencias entre los participantes con menor edad y los de 15 y 16 años, y $d=.20$, para las diferencias entre los participantes con menor edad y los de 17 y 18 años) y Verbal $(d=.16$, para las diferencias entre los participantes con menor edad y los de 15 y 16 años, y $d=.23$, para las diferencias entre los participantes con menor edad y los de 17 y 18 años).

No se encontró un efecto estadísticamente significativo para la interacción género por edad en las 11 dimensiones del SDQII-S.

Tabla 3

Diferencias según el género

\begin{tabular}{|c|c|c|c|c|c|c|c|}
\hline & \multicolumn{2}{|c|}{ Varones } & \multicolumn{2}{|c|}{ Mujeres } & \multicolumn{3}{|c|}{$\begin{array}{l}\text { Significación estadística y } \\
\text { magnitud de las diferencias }\end{array}$} \\
\hline & $M$ & $D E$ & $M$ & $D E$ & $F_{(1,1412)}$ & $p$ & $d$ \\
\hline Académico General & 16.48 & 4.37 & 16.32 & 4.46 & .02 & .87 & - \\
\hline Matemáticas & 13.62 & 5.49 & 12.01 & 5.66 & 25.44 & .00 & .29 \\
\hline Verbal & 17.67 & 5.77 & 19.03 & 5.82 & 22.26 & .00 & .23 \\
\hline Apariencia Física & 13.99 & 4.63 & 14.59 & 4.95 & 6.32 & .01 & .13 \\
\hline Habilidades Físicas & 17.79 & 4.83 & 16.72 & 5.64 & 15.33 & .00 & .20 \\
\hline Estabilidad Emocional & 17.56 & 4.67 & 14.75 & 4.85 & 109.74 & .00 & .59 \\
\hline Relación con los Padres & 19.27 & 4.27 & 18.58 & 4.86 & 9.01 & .00 & .15 \\
\hline Relaciones con el Sexo Opuesto & 17.07 & 4.04 & 16.98 & 3.89 & .61 & .43 & - \\
\hline Relaciones con el Mismo Sexo & 22.66 & 4.70 & 22.30 & 4.76 & 1.96 & .16 & - \\
\hline Sinceridad/Veracidad & 25.81 & 5.30 & 27.38 & 5.44 & 22.71 & .00 & .29 \\
\hline Autoestima & 26.95 & 5.31 & 27.75 & 5.16 & 6.85 & .00 & .15 \\
\hline
\end{tabular}


Tabla 4

Diferencias según la edad

\begin{tabular}{lcccccccc}
\hline & \multicolumn{2}{c}{$13-14$ años } & \multicolumn{2}{c}{$15-16$ años } & \multicolumn{2}{c}{$17-18$ años } & \multicolumn{2}{c}{$\begin{array}{c}\text { Significación } \\
\text { estadística }\end{array}$} \\
\cline { 2 - 9 } & \multicolumn{1}{c}{ A } & $D E$ & $M$ & $D E$ & $M$ & $D E$ & $F_{(2,1411)}$ & $p$ \\
\cline { 2 - 9 } Académico General & 16.18 & 4.36 & 16.18 & 4.45 & 17.01 & 4.37 & 4.51 & .01 \\
Matemáticas & 13.49 & 5.18 & 12.60 & 5.73 & 12.37 & 5.91 & 4.16 & .01 \\
Verbal & 19.12 & 5.67 & 18.18 & 5.95 & 17.83 & 5.75 & 6.01 & .00 \\
Apariencia Física & 13.58 & 4.69 & 14.19 & 4.96 & 15.28 & 4.50 & 12.44 & .00 \\
Habilidades Físicas & 16.67 & 5.19 & 16.91 & 5.36 & 18.46 & 5.07 & 14.10 & .00 \\
Estabilidad Emocional & 16.98 & 4.98 & 15.89 & 4.99 & 15.58 & 4.79 & 8.47 & .00 \\
Relación con los Padres & 19.07 & 4.64 & 18.83 & 4.59 & 18.91 & 4.56 & .28 & .75 \\
Relaciones con el Sexo & 16.64 & 3.88 & 17.08 & 3.99 & 17.35 & 3.99 & 3.25 & .03 \\
Opuesto & & & & & & & & \\
Relaciones con el Mismo & 22.36 & 4.55 & 22.52 & 4.71 & 22.53 & 4.98 & .19 & .82 \\
Sexo & & & & & & & & .62 \\
Sinceridad/Veracidad & 26.62 & 5.11 & 26.75 & 5.56 & 26.37 & 5.55 & .46 & .62 \\
Autoestima & 26.47 & 5.69 & 27.28 & 5.14 & 28.48 & 4.69 & 14.63 & .00 \\
\hline
\end{tabular}

\section{Discusión y conclusión}

El presente estudio tuvo por objeto analizar las diferencias de género y edad en las dimensiones del autoconcepto, evaluadas mediante el SDQII-S, siendo el primer estudio de estas características realizado con población adolescente chilena.

En relación con las diferencias de género, los resultados apoyaron parcialmente nuestra hipótesis inicial. Por un lado, y en consonancia con investigaciones previas (Chu, 2002; Inglés et al., 2009; Nishikawa et al., 2007; Yun, 2001), se halló que las mujeres obtuvieron una media significativamente superior a los hombres en el Autoconcepto Verbal y en la dimensión de Sinceridad/Veracidad. Asimismo, se encontró que los hombres, puntuaron más alto en las dimensiones de Matemáticas (Chu, 2002; Inglés et al., 2009; Yun, 2001), Habilidades Físicas y Estabilidad Emocional (Chu,
2002; Hay, 2000; Inglés et al., 2009). Además, apoyando los hallazgos de Hay (2000) e Inglés et al. (2009), no se obtuvieron diferencias entre ambos sexos para la variable Relaciones con el Sexo Opuesto, ni tampoco para Relaciones con el Mismo Sexo, en la línea de Hay (2000) y Nishikawa et al. (2007), así como para la dimensión Autoconcepto Académico General, en consonancia con Inglés et al. (2009), Nishikawa et al. (2007) y Yun (2001) y contradiciendo los trabajos de Hay (2000) y Chu (2002) quienes reportaron mayores niveles para los varones. $\mathrm{Ca}-$ be recordar, igualmente, que todos estos trabajos fueron realizados empleando población general adolescente, en consonancia con el presente estudio, con excepción de las investigaciones de Hay (2000) y Yun (2001) quienes utilizaron, respectivamente, participantes con conductas disruptivas y con altas capacidades, además de alumnado asistente a centros regulares. 
Contrariamente a lo esperado, los varones obtuvieron mayores puntuaciones en Relación con los Padres que sus iguales del sexo opuesto, y a la inversa en las dimensiones de Autoestima y Apariencia Física, contradiciendo los trabajos realizados por $\mathrm{Chu}$ (2002), Hay (2000), Inglés et al. (2009) y Nishikawa et al. (2007). Llama la atención el hecho de que los varones tiendan a percibirse a sí mismos como menos atractivos que las mujeres, puesto que no es consistente con la literatura existente, que ha evidenciado que las adolescentes tienden a reportar una mayor insatisfacción corporal que sus compañeros, así como una mayor probabilidad de desarrollar trastornos de la conducta alimentaria (Ferreiro, Seoane y Serna, 2014). Sin embargo, recientemente, se ha evidenciado que la preocupación por el cuerpo y el deseo de desarrollar la musculatura es cada vez más prevalente en la población masculina, probablemente debido a un aumento de la presión social en la apariencia de los hombres (véase McCabe y Ricciardelli, 2004, y Blond, 2008, para una revisión). Respecto de la dimensión Relación con los Padres, es conveniente tener presentes las diferencias encontradas, pues se ha demostrado que la percepción acerca del vínculo familiar influye en el bienestar y la resiliencia de los adolescentes (Leiva, Pineda y Encina, 2013).

Cabe mencionar, que los tamaños del efecto fueron pequeños en todos los casos, con excepción de un efecto moderado hallado en las diferencias de género para la variable Estabilidad Emocional. Este resultado se explica por el hecho de que existen claras diferencias de género en la salud percibida durante la adolescencia, puesto que los cambios puberales influyen negativamente en las mujeres, de manera que tienden a percibir peor su salud, tanto física como emocional (Benjet y Hernández-Guzmán, 2002; VélezGalárraga, López-Aguilà y Rajmil, 2009).
Desde el punto de vista académico, destacan las diferencias sexuales encontradas en las variables de Autoconcepto Matemático y Verbal, acordes con los resultados obtenidos por los estudiantes chilenos en las áreas de Comprensión Lectora y Matemáticas del Informe PISA 2012, donde se advierte la existencia de la mayor brecha de género del continente americano (Gobierno de Chile, 2013). En este sentido, teniendo en consideración que, tal y como ha evidenciado la literatura científica, el autoconcepto es un importante predictor del rendimiento y el ajuste académico (Guay, Pantano y Boivin, 2003; Guterman, Hahm y Cameron, 2002; Musitu, Buelga, Lila y Cava, 2001), investigaciones futuras deberían centrarse en desarrollar programas de intervención destinados a fomentar el Autoconcepto Verbal y en Matemáticas de los adolescentes chilenos y a minimizar las diferencias existentes en función del sexo.

En relación con las diferencias de edad, los resultados mostraron una tendencia de los estudiantes de mayor edad a obtener puntuaciones más altas que sus iguales de menor edad en las dimensiones de Autoconcepto Académico General, Apariencia Física, Habilidades Físicas, Relaciones con el Sexo Opuesto y Autoestima. Inversamente, se observó un descenso de las puntuaciones en Matemáticas, Verbal y Estabilidad Emocional. Esta tendencia a reportar un menor autoconcepto en las dos áreas académicas en las edades de 17 y 18 años puede deberse al incremento de dificultad y de las exigencias en los cursos superiores. Por otro lado, en lo que respecta a la Estabilidad Emocional, la última Encuesta Nacional de Salud (Ministerio de Salud de Chile, 2009) realizada a mayores de 15 años, establece que Chile es un país con altas tasas de enfermedades mentales y algunos autores advierten que se trata de un problema en aumento que no está reci- 
biendo la atención necesaria (Leiva et al., 2015). Por su parte, Hanquin, Larraguibel y Cabezas (2004) encontraron que los bajos niveles de autoconcepto y autoestima eran el principal factor de riesgo para la salud mental de los adolescentes chilenos. Por tanto, sería conveniente llevar a cabo estrategias que fomentasen la percepción que estos tienen acerca de su ajuste emocional, con el objetivo de prevenir problemas mentales y su consecuente repercusión en el coste económico del país.

No se hallaron diferencias según la edad para las dimensiones de Relaciones con los Padres, Relaciones con el Mismo Sexo y Sinceridad/Veracidad, al igual que en el estudio realizado por Chu (2002).

Es preciso señalar que el presente trabajo posee una serie de limitaciones. En primer lugar, puesto que se trata de un diseño transversal, no podemos evaluar los perfiles de cambio intrasujetos, aspecto que podría abordarse en el futuro median- te estudios longitudinales. Asimismo, ya que se ha utilizado una única medida de autoinforme para evaluar el autoconcepto, sería conveniente que en el futuro se emplearan distintos instrumentos para la recolección de datos, como por ejemplo entrevistas de respuesta abierta o completadas por padres y profesores. Igualmente, sería conveniente ampliar el rango de edad, incluyendo estudiantes a partir de los 11 años, con el objetivo de tener una muestra más representativa de la adolescencia. Por último, futuros trabajos podrían comparar las puntuaciones medias reportadas por los adolescentes chilenos con la media teórica de cada una de las dimensiones del autoconcepto o con las puntuaciones medias obtenidas por adolescentes de otros países. Pese a ello, este es el primer estudio que analiza las diferencias de género y edad en el autoconcepto, empleando población adolescente chilena, siendo, por tanto, un aporte novedoso para el campo del estudio del autoconcepto en Chile.

\section{Referencias}

Amezcua, J. A. y Pichardo, M. C. (2000). Diferencias de género en autoconcepto en sujetos adolescentes. Anales de Psicología, 16(2), 207-214.

Benjet, C. \& Hernández-Guzmán, L. (2002). A short-term longitudinal study of pubertal change, gender, and psychological well-being of Mexican early adolescents. Journal of Youth and Adolescence, 31(6), 429-442.

http://dx.doi.org/10.1023/A:1020259019866

Blond, A. (2008). Impacts of exposure to images of idea bodies on male body dissatisfaction: A review. Body Image, 5(3), 244-250.

http://dx.doi.org/10.1016/j.bodyim.2008.02.003

Bodkin-Andrews, G. H., Hay, M. T., Craven, R. G., \& Yeung, A. S. (2010). Factorial invariance testing and latent mean differences for the Self-Description Questionnaire II (Short Version) with indigenous and non-indigenous Australian secondary school students. International Journal of Testing, 10(1), 47-79.

http://dx.doi.org/10.1080/15305050903352065

Bodkin-Andrews, G. H., O’Rourke, V., Dillon, A., Graven, R. G., \& Seeshing-Yeung, A. (2012). Engaging the disengaged?: A longitudinal analysis of the relations between in- 
digenous and non-indigenous Australian students' academic self-concept and disengagement. Journal of Cognitive Education and Psychology, 11(2), 179-195.

http://dx.doi.org/10.1891/1945-8959.11.2.179

Cardenal, V. y Díaz, J. F. (2000). Diferencias según el sexo en autoestima y en su asociación con otras variables psicológicas en adolescentes. Revista de Psicología Educativa, 6(2), 105-128.

Chu, Y. W. (2002). The relationships between domain-specific self-concepts and global selfesteem among adolescents in Taiwan. Bulletin of Educational Psychology, 33, 103-124.

Cohen, J. (1988). Statistical power analysis for the behavioral sciences (2nd Ed.). Hillsdale, NJ: Erlbaum.

Cunningham, L. G. \& Rinn, A. N. (2007). The role of gender and previous participation in a summer program on gifted adolescents' self-concepts over time. Journal for the Education of the Gifted, 30(3), 326-416.

http://dx.doi.org/10.1177/016235320703000303

Delgado, B., Inglés, C. J., \& García-Fernández, J. M. (2013). Social anxiety and selfconcept in adolescence. Revista de Psicodidáctica, 18(1), 179-194.

http://dx.doi.org/10.1387/RevPsicodidact.6411

Ferreiro, F., Seoane, G., \& Serna, C. (2014). Toward understanding the role of body dissatisfaction in the gender differences in depressive symptoms and disorders eating: A longitudinal study during adolescence. Journal of Adolescence, 37, 73-84.

http://dx.doi.org/10.1016/j.adolescence.2013.10.013

Garaigordobil, M., Durá, A., \& Pérez, J. I. (2005). Psychopathological symptoms, behavioral problems, and self-concept, self-esteem: A study with adolescents from 14 to 17 years. Annuary of Clinical and Health Psychology, 1, 53-63.

García-Fernández, J. M., Torregrosa, M. S., Inglés, C. J., Ruiz-Esteban, C., Pastor, Y., Martínez-Zaragoza, F., ... Delgado, B. (julio, 2006). Analysis of the self-concept dimensionality in a Spanish sample of secondary school students. Trabajo presentado en el $26^{\text {th }}$ Congress of Applied Psychology, Atenas.

Gobierno de Chile, Agencia de la Calidad de la Educación y Organización para la Cooperación y el Desarrollo Económicos. (2013). Resultados PISA 2012 Chile. Recuperado de https://s3-us-west-2.amazonaws.com/documentosweb/Informes/Resultados+PISA+2012+Chile.pdf

Gómez-Vela, M., Verdugo, M. A. y González-Gil, F. (2007). Calidad de vida y autoconcepto en adolescentes con necesidades educativas especiales y sin ellas. Infancia y Aprendizaje, 30(4), 523-536.

http://dx.doi.org/10.1174/021037007782334300

Gordillo, M., Vicente, F., Sánchez, S., Gómez, M. y Gordillo, T. (2011). Diferencias en el autoconcepto de adolescentes extremeños en función del género, el nivel socioeconómico-cultural y la población de pertenencia. Campo Abierto, 30(2), 65-78.

Guay, F., Pantano, H. y Boivin, M. (2003). Autoconcepto y logro académico: perspectivas del desarrollo sobre su ordenamiento causal. Diario de Psicología Educativa, 95(2), 124-136. 
Guido, P., Mujica, A. y Gutiérrez, R. (2011). Diferencias en el autoconcepto por sexo en la adolescencia: construcción y validación de un instrumento. Liberabit, 17(2), 139-146.

Grum, K. (2006). Does mental health promoting school program (in central and eastern European schools) improve self-concept? Studia Psychologica, 48(2), 125-140.

Guterman, N. B., Hahm, H. C., \& Cameron, M. (2002). Adolescent victimization and subsequent use of mental health counselling services. Journal of Adolescent Health, 30, 336-345. http://dx.doi.org/10.1016/S1054-139X(01)00406-2

Hanquin, C., Larraguibel, M. y Cabezas, A. (2004). Factores protectores y de riesgo en salud mental en niños y adolescentes de la ciudad de Calama. Revista Chilena de Pediatría, 75(5), 425-433.

Hay, I. (2000). Gender self-concept profiles of adolescents suspended from high school. Journal of Child Psychology and Psychiatry, 41(3), 345-352.

http://dx.doi.org/10.1111/1469-7610.00618

Huebner, E. S., Gilman, R., \& Laughlin, J. E. (1999). A multimethod investigation of the multidimensionality of children's well-being reports: Discriminant validity of life satisfaction and self-esteem. Social Indicators Research, 46(1), 1-22.

Infante, L., De la Morena, L., García, B., Sánchez, A., Hierrezuelo, L. y Muñoz, A. (2002). Un estudio sobre el autoconcepto social en estudiantes de ESO: diferencias de género. Revista Electrónica Interuniversitaria de Formación del Profesorado, 5(3), 1-7.

Inglés, C., Martínez-González, A., García-Fernández, J. M., Torregrosa, M. S., \& RuizEsteban, C. (2012). Prosocial behavior and self-concept of Spanish students of compulsory secondary education. Revista de Psicodidáctica, 17(1), 135-156.

http://dx.doi.org/10.1387/RevPsicodidact.1861

Inglés, C., Pastor, Y., Torregrosa, M. S. Redondo, J. y García-Fernández, J. M. (2009). Diferencias en función del género y el curso académico en dimensiones del autoconcepto: estudio con una muestra de adolescentes españoles. Anuario de Psicología, 40(2), 271-288.

Lagos-San Martín, N., García-Fernández, J. M., Inglés, C. J., Gómez-Núñez, M. I. e Hidalgo, M. D. (en prensa). Self-Description Questionnaire II (versión breve): evidencia de fiabilidad y validez en una muestra de adolescentes chilenos. Revista Latinoamericana de Psicología.

Leiva, L., George, M., Antivilo, A., Squicciarini, A., Simonsohn, A., Vargas, B. y Guzmán, J. (2015). Salud mental escolar: logros de una intervención preventiva en salud mental en niños y niñas del primer ciclo de enseñanza básica. Psicoperspectivas, 14(1), 31-41. Recuperado de http://www.psicoperspectivas.cl/index.php/psicoperspectivas/article/viewFile/508/402

Leiva, L., Pineda, M. y Encina, Y. (2013). Autoestima y apoyo social como predictores de la resiliencia en un grupo de adolescentes en vulnerabilidad social. Revista de Psicología, 22(2), 111-123.

http://dx.doi.org/10.5354/0719-0581.2013.30859

Marsh, H. W. (1989). Age and sex effects in multiple dimensions of self-concept: Preadolescence to early adulthood. Journal of Educational Psychology, 81, 417-430. http://dx.doi.org/10.1037/0022-0663.81.3.417 
Marsh, H. W. (1992). Self-Description Questionnaire (SDQ) II: A theoretical and empirical basis for the measurement of multiple dimensions of adolescent self-concept: An interim test manual and a research monograph. New South Wales: University of Western Sydney.

Marsh, H. W., Ellis, L. A., Parada, R. H., Richards, G., \& Heubeck, B. G. (2005). A short version of the Self-Description Questionnaire II: Operationalizing criteria for short-form evaluation with new applications of confirmatory factor analyses. Psychological Assessment, 17(1), 81-102.

http://dx.doi.org/10.1037/1040-3590.17.1.81

Marsh, H. W. \& Hau, K. T. (2004). Explaining paradoxical relations between academic selfconcepts and achievements: Cross-cultural generalizability of the internal-external frame of reference predictions across 26 countries. Journal of Educational Psychology, 96, 56-67. http://dx.doi.org/10.1037/0022-0663.96.1.56

Marsh, H. W., Martin, A. J., \& Jackson, S. (2010). Introducing a short version of the Physical Self-Description Questionnaire: New strategies, short-form evaluative criteria, and applications of factor analyses. Journal of Sport y Exercise Psychology, 32, 438-482.

Marsh, H. W., Parada, R. H., \& Ayotte, V. (2004). A multidimensional perspective of relations between self-concept (Self-Description Questionnaire II) and adolescent mental health (Youth Self-Report). Psychological Assessment, 16(1), 27-41.

http://dx.doi.org/10.1037/1040-3590.16.1.27

Massanero, M. A. y Vázquez, A. (2005). Autoconcepto y rendimiento escolar: relaciones con otras variables psicopedagógicas. Educació i Cultura, 18, 143-165.

McCabe, M. P. \& Ricciardelli, L. A. (2004). Body image dissatisfaction among males across the lifespan. A review of past literature. Journal of Psychosomatic Research, 56, 675-685. http://dx.doi.org/10.1016/S0022-3999(03)00129-6

Ministerio de Salud de Chile. (2009). Encuesta Nacional de Salud ENS Chile 2009-2010. Recuperado de http://web.minsal.cl/portal/url/item/bcb03d7bc28b64dfe040010165012d23.pdf

Musitu, G., Buelga, S., Lila, M. y Cava, M. J. (2001). Familia y adolescencia: un modelo de análisis e intervención psicosocial. Madrid: Síntesis.

Nishikawa, S., Hägglöf, B., \& Sundbom, E. (2010). Contributions of attachment and selfconcept on internalizing and externalizing problems among Japanese adolescents. Journal of Child and Family Studies, 19(3), 334-342.

http://dx.doi.org/10.1007/s10826-009-9303-9

Nishikawa, S., Norlander, T., Fransson, P., \& Sundbom, E. (2007). A cross-cultural validation of adolescent self-concept in two cultures: Japan and Sweden. Social Behavior and Personality, 35(2), 269-286.

http://dx.doi.org/10.2224/sbp.2007.35.2.269

Nishikawa, S., Sundbom, E., \& Hägglöf, B. (2010). Influence of perceived parental rearing on adolescent self-concept and internalizing and externalizing problems in Japan. Journal of Child and Family Studies, 19, 57-66.

http://dx.doi.org/10.1007/s10826-009-9281-y 
Padilla, M. T., García, S. y Suárez, M. (2010). Diferencias de género en el autoconcepto general y académico de estudiantes de $4^{\circ}$ de ESO. Revista de Educación, 352, 495-515.

Palenzuela, D. (1983). Construcción y validación de una escala de autoeficacia percibida específica de situaciones académicas. Análisis y Modificación de Conducta, 9, 185-219.

Pastor, Y., Balaguer, I. y García-Merita, M. L. (2003). El autoconcepto y la autoestima en la adolescencia media: análisis diferencial por curso y género. Revista de Psicología Social, 18(2), 141-159.

http://dx.doi.org/10.1174/021347403321645258

Razali, A., Razali, N. A., Azizan, C. R., \& Pillai, R. (2014). Self-concept: Differences among Malay adolescents by gender. Asian Social Science, 10(1), 265-275. http://dx.doi.org/10.5539/ass.v10n1p265

Rigoli, D., Piek, J. P., \& Kane, R. (2012). Motor coordination and psychosocial correlates in a normative adolescent sample. Pediatrics, 129(4), 892-900.

http://dx.doi.org/10.1542/peds.2011-1237

Shavelson, J., Hubner, J. J., \& Stanton, G. C. (1976). Self-concept: Validation of construct interpretations. Review of Educational Research, 46, 407-442.

http://dx.doi.org/10.3102/00346543046003407

Simmons, N. \& Hay, I. (2010). Early adolescents' friendship patterns in middle school: Social-emotional and academic implications. The Australian Educational and Developmental Psychologist, 27(2), 59-69.

http://dx.doi.org/10.1375/aedp.27.2.59

Spielberger, C. D., Gorsuch, R. L., \& Lushene, R. E. (1970). Manual for the State/Trait Anxiety Inventory. Palo Alto, CA: Consulting Psychologists Press.

Vasconcelos-Rasposo, J., Gonçalves, O., Teixeira, C., \& Fernandes, H. M. (2009). Relação entre dimensões do autoconcepto e consume de álcool em alunos. Motricidade, 5(1), 51-76.

Vélez-Galárraga, R., López-Aguilà, S. y Rajmil, L. (2009). Género y salud percibida en la infancia y la adolescencia en España. Gaceta Sanitaria 23(5), 433-439.

http://dx.doi.org/10.1016/j.gaceta.2009.01.014

Yun, D. (2001). A comparison of gender differences in academic self-concept and motivation between high ability and average Chinese adolescents. Journal of Secondary Gifted Education, 13(1), 22-32.

http://dx.doi.org/10.4219/jsge-2001-361 